\title{
La spectrométrie de masse par accélérateur
}

\section{Une technique de physique nucléaire appliquée à la reconstitution des variations passées du champ magnétique terrestre}

Didier L. Bourlés (bourles@cerege.fr), Quentin Simon et Nicolas Thouveny

CEREGE (UMR7330, Aix-Marseille Université, CNRS, IRD, INRA, Collège de France)

Technopole Environnement Arbois-Méditerranée, BP 80, 13545 Aix-en-Provence Cedex 4

L'étude des nucléides

cosmogéniques et leur application

aux géosciences débutent

dans les années 1980,

avec le développement

de spectromètres de masse

par accélération dédiés.

Depuis 2007, grâce à l'instrument

national ASTER (Accélérateur

pour les Sciences de la Terre,

Environnement, Risques)

du CEREGE, des progrès majeurs

ont été réalisés. Ils ont permis

la quantification jusqu'alors

impossible de nombreux

processus géophysiques.

Parmi ces avancées, la restitution

fidèle du champ magnétique

terrestre pendant le dernier

million d'années a été rendu

possible par les mesures de concentration du béryllium-10

$\left({ }^{10} \mathrm{Be}\right)$ atmosphérique.
Le rayonnement cosmique primaire arrivant au sommet de l'atmosphère contient, en nombre, 99\% de particules chargées positivement - protons (p) et noyaux d'hélium $(\alpha)$ - et 1\% de noyaux lourds et d'électrons. Au sein de ce rayonnement, la plupart des particules les plus énergétiques (d'environ $10^{15}$ à $10^{21}$ électronvolts $(\mathrm{eV})$ ) ont une origine extragalactique, la majorité de celles dont l'énergie est comprise entre $\sim 10^{10}$ et $\sim 10^{15} \mathrm{eV}$ est d'origine galactique, tandis que les particules les moins énergétiques $\left(\leq 10^{10} \mathrm{eV}\right)$ sont essentiellement produites par le Soleil. Les composantes solaire et galactique different aussi par la variation de leur composition et de leur intensité au cours du temps [1]. À l'approche de la Terre, ces particules primaires interferent avec la magnétosphère qui agit comme un bouclier contre le bombardement de particules chargées. Ce rôle protecteur a été particulièrement important pour l'apparition et la préservation de la vie, et est primordial pour le fonctionnement des instruments placés en orbite. La pérennité et le maintien futur de la magnétosphère sont cependant mis en doute. En effet, les observations confirment une décroissance continue de l'intensité du champ magnétique terrestre mesurée depuis le début du XIX ${ }^{\mathrm{e}}$ siècle, et mettent en évidence des anomalies telles que celle de la région Atlantique $\mathrm{Sud}^{(\mathrm{a})}$. Dans ce contexte, il s'avère indispensable de reconstruire les variations de ce champ à l'échelle des derniers millions d'années, en couvrant ainsi des évènements tels que les inversions ${ }^{(b)}$ et les excursions ${ }^{(c)}$, afin de caractériser les perturbations du fonctionnement de la géodynamo impliquées, et tenter d'anticiper son évolution au cours des prochains siècles.

\section{Les nucléides cosmogéniques}

On appelle «nucléides cosmogéniques » les noyaux atomiques générés dans l'atmosphère ou dans les premiers mètres de la croute terrestre par des réactions de spallation nucléaires (fig. 1) initiées par les particules du rayonnement cosmique. Lors de chaque réaction nucléaire, une partie de l'énergie initiale des particules incidentes (appelées particules "primaires ») est dissipée et transférée lors de collisions élastiques aux constituants (protons et neutrons) du noyau impacté. Ainsi, l'énergie des particules primaires diminue lors de leur traversée de l'atmosphère, jusqu'à devenir insuffisante pour induire des réactions nucléaires (lorsque l'énergie de la particule est inférieure à l'énergie seuil de la réaction nucléaire). Tout comme pour les particules primaires, l'énergie des particules dites "secondaires" (essentiellement des neutrons) émises lors de la réaction initiale diminue également lors de leur traversée de l'atmosphère, jusqu'à devenir insuffisante pour induire des réactions nucléaires.

En conséquence, l'essentiel des réactions nucléaires conduisant à la production de nucléides cosmogéniques se produit dans la stratosphère, entre 12 et 50 km d'altitude en moyenne. Une particule primaire ou secondaire sur mille seulement atteint la surface terrestre avec suffisamment d'énergie pour y induire des réactions de spallation 


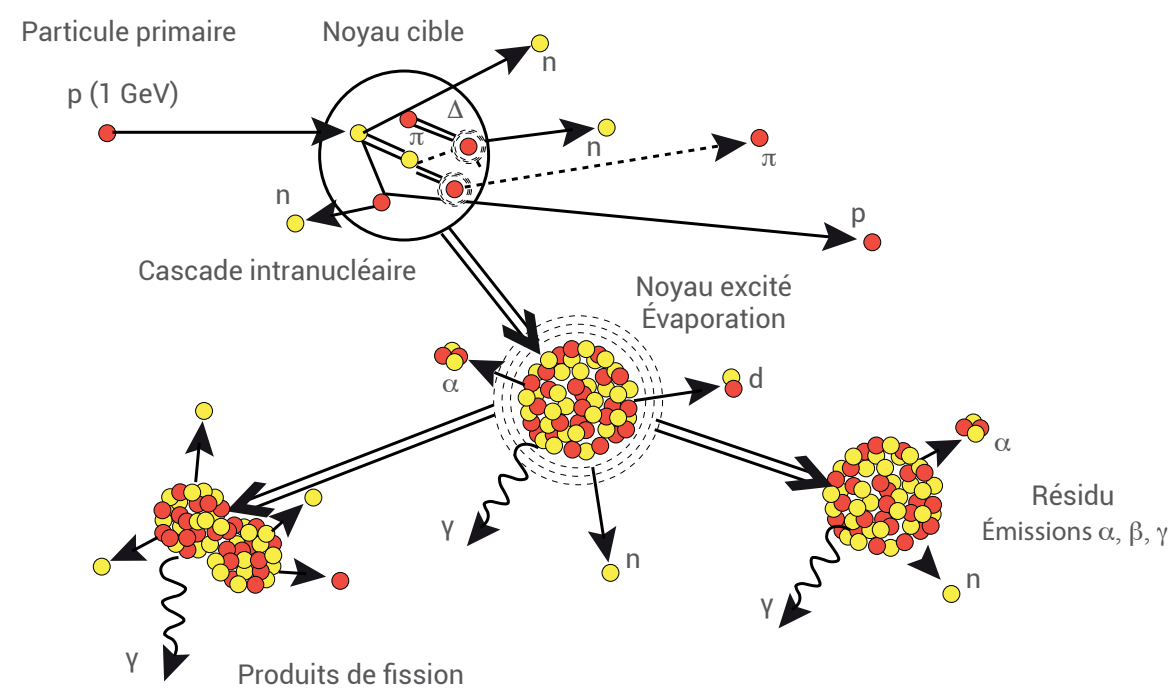

avec les éléments constitutifs des minéraux des roches, produisant les nucléides cosmogéniques dits in situ, dont les concentrations sont très faibles relativement à celles des nucléides cosmogéniques produits dans l'atmosphère.

En résumé, ces réactions laissent comme résidus des noyaux d'une espèce chimique différente de l'espèce initiale, puisque le numéro atomique (nombre de protons) et le nombre de masse (nombre de protons + nombre de neutrons) sont inférieurs à ceux du noyau cible originel. Parmi les nucléides cosmogéniques ainsi produits, les six isotopes les plus communément utilisés en géosciences sont l'hélium 3 (stable), le béryllium 10 (de période radioactive $\mathrm{T}_{1 / 2}=(1,387 \pm 0,012) \cdot 10^{6}$ ans $)$, le carbone $14\left(\mathrm{~T}_{1 / 2}=(5734 \pm 40)\right.$ ans $)$, le néon 21 (stable), l'aluminium 26 $\left(\mathrm{T}_{1 / 2}=(7,17 \pm 0,17) \cdot 10^{5}\right.$ ans) et le chlore $36\left(\mathrm{~T}_{1 / 2}=(3,01 \pm 0,02) \cdot 10^{5}\right.$ ans $)$. Le béryllium 10 atmosphérique, par exemple, est produit à partir des deux principaux constituants de l'atmosphère que sont l'azote 14 et l'oxygène 16 à travers les réactions de spallation :

${ }_{7}^{14} \mathrm{~N}+{ }_{0}^{1} \mathrm{n} \rightarrow{ }_{4}^{10} \mathrm{Be}_{\mathrm{atm}}+3{ }_{1}^{1} \mathrm{p}+{ }_{0}^{1} \mathrm{n}$ et ${ }_{8}^{16} \mathrm{O}+{ }_{0}^{1} \mathrm{n} \rightarrow{ }_{4}^{10} \mathrm{Be}_{\mathrm{atm}}+4{ }_{1}^{1} \mathrm{p}+3{ }_{0}^{1} \mathrm{n}$.

L'étude de la concentration des nucléides cosmogéniques produits dans l'atmosphère ou préservés dans les archives géologiques (sédiments marins et lacustres, carottes de glace, nodules de manganèse...), permet donc de reconstituer les modulations passées du flux de rayonnement cosmique incident. Ces modulations sont induites par la variabilité des sources de rayonnement

1. Schéma d'une réaction de spallation nucléaire. Cette réaction comporte deux étapes. Lors de la première étape, très courte (quelques $10^{-22} \mathrm{~s}$ ), la particule incidente de haute énergie entre en collision avec les nucléons du noyau-cible. Une cascade intranucléaire se développe, et des protons $(p)$, neutrons $(n)$ et pions $(\pi)$ rapides sont émis. Le noyau-cible est alors un noyau hautement excité qui, lors de la seconde étape (beaucoup plus lente) de la réaction, peut soit se désexciter en émettant des neutrons et des particules $\alpha, \beta, \gamma$, soit se fragmenter en plusieurs produits de fission. (Source : Irfu, CEA/Saclay).

elles-mêmes (considérée comme négligeable à l'exception d'évènements ponctuels identifiables comme l'explosion d'une supernova, par exemple) et, surtout, par les variations d'intensité des champs magnétiques solaire et terrestre qui contribuent à dévier les particules chargées du rayonnement cosmique primaire (via la force de Lorentz). Les nucléides cosmogéniques préservés avec une résolution quasi annuelle dans les couches de glace résultant des chutes de neige hivernales, permettent par conséquent de reconstituer les variations de l'activité solaire caractérisées principalement par de hautes fréquences, alors que l'influence du champ magnétique terrestre se démarque par de plus larges amplitudes de production et des échelles de temps centenaires à millénaires (voir p. 20). Par ailleurs, ces variations de production offrent un outil fiable de corrélation stratigraphique entre des archives géologiques distinctes réparties à travers le globe terrestre.

La désintégration des nucléides cosmogéniques radioactifs est également un outil de datation absolue remarquable, contraint par le temps de demi-vie des éléments étudiés. À cet égard, le carbone 14 atmosphérique est certainement le nucléide le plus utilisé, permettant de remonter jusqu'à 40000 ans, dans des domaines variés allant de la paléoclimatologie à l'archéologie, notamment. Jusqu'à 10 à 15 millions d'années, le béryllium 10 atmosphérique est particulièrement bien adapté pour dater les dépôts sédimentaires dans lesquels il s'accumule. Il a par exemple permis de dater l'apparition des premiers hominidés au Tchad, il y a 7 millions d'années [2].

Contrairement à celles des nucléides cosmogéniques atmosphériques, les concentrations des nucléides cosmogéniques produits in situ sont principalement liées à l'historique d'exposition de l'échantillon au rayonnement cosmique. Leur étude permet donc de quantifier, par exemple : i/ les déformations tectoniques et donc de préciser l'aléa sismique ; ii/ les paléoclimats continentaux ; iii/ les interactions entre l'érosion, le climat et la tectonique ; iv/ les processus de mise en place et d'évolution des surfaces continentales ; v/ le développement des profils d'altération ; vi/ les taux de dénudation à l'échelle de bassins-versants, etc. Ils offrent dès lors l'opportunité d'une étude cinétique, dynamique et temporelle des processus géomorphologiques et paléoclimatiques affectant la surface terrestre. 
2. Vue panoramique de l'instrument national spectromètre de masse par accélérateur ASTER (Accélérateur pour les Sciences de la Terre, Environnement, Risques), schéma des principaux éléments et représentation des différentes trajectoires suivies par les isotopes et isobares.

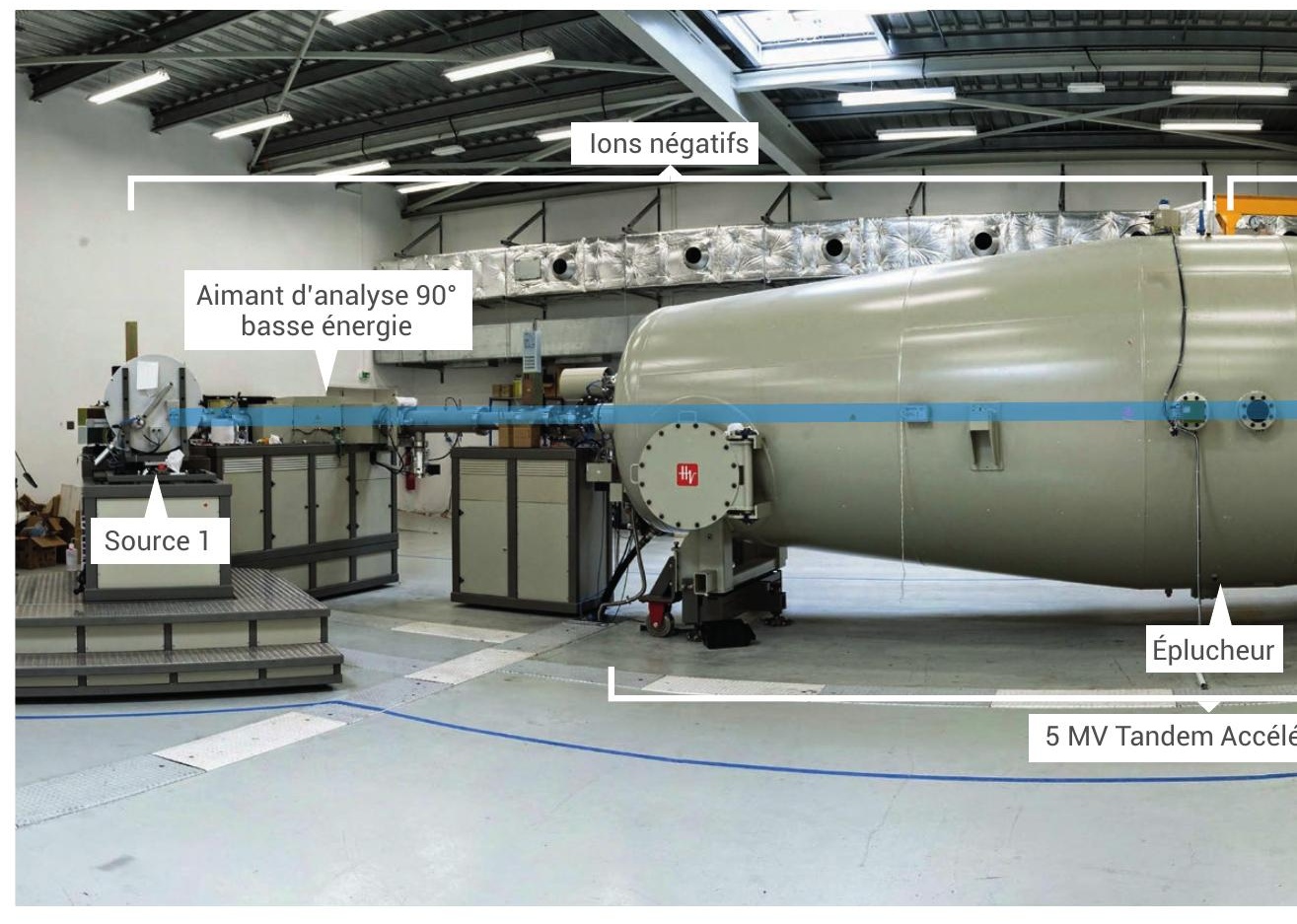

cosmogéniques a dès lors motivé le développement de la technique de spectrométrie de masse par accélérateur.

Pour identifier, détecter et compter les atomes d'un isotope d'un élément chimique donné, il faut déterminer à la fois son nombre de masse et son numéro atomique (nombre de charge). Au nombre de masse correspond pour une énergie et un état de charge donnés un seul rayon de courbure de la trajectoire dans un champ magnétique donné ; au numéro atomique correspond une perte d'énergie par dissipation spécifique dans une épaisseur de matière donnée. L'utilisation de cette dernière caractéristique, suivie de la détection et du comptage de l'atome à analyser, n'est possible que si l'énergie des deux isobares est suffisamment élevée pour : (i) autoriser une perte d'énergie différentielle substantielle et (ii) préserver une énergie résiduelle permettant de les transporter jusqu'à et à travers un aimant d'analyse afin de les séparer efficacement. La spectrométrie de masse par accélérateur, réalisée par exemple sur l'instrument ASTER (encadré 1 et figure 2), introduit donc un étage d'accélération entre les parties source et analyse d'un spectromètre de masse classique, afin de procurer cette énergie nécessaire.

Une des avancées les plus remarquables liée au développement de cette technique d'analyse est l'amélioration par un facteur un million à un milliard de la sensibilité de détection par rapport aux techniques classiques (spectrométrie de masse conventionnelle, comptage des décroissances radioactives). Grâce à la spectrométrie de masse par accélérateur, il est désormais possible :

- de permettre des études à haute résolution sur des quantités de matière faibles (0,1 g de sédiment marin, par exemple) ; - de dater plus précisément à partir de faibles quantités de différents types de substrat sur une gamme temporelle $\left(\sim 10\right.$ périodes $\left.T_{1 / 2}\right)$ significativement $(2$ à 3 fois) plus étendue que celle accessible auparavant ;

- de réduire à quelques minutes la durée nécessaire à l'acquisition des résultats, durée qui était auparavant de plusieurs heures ;

- de simplifier les manipulations physiques et chimiques lors de la préparation des échantillons pour leur analyse.

\section{Application au champ magnétique terrestre}

Le champ magnétique terrestre (CMT) est entretenu par le brassage du fer liquide du noyau externe sous l'effet des convections d'origine thermique et compositionnelle et de la rotation de la Terre. Les changements de régime de cette dynamo auto-excitée et de ses interactions génèrent des variations temporelles du CMT sur 


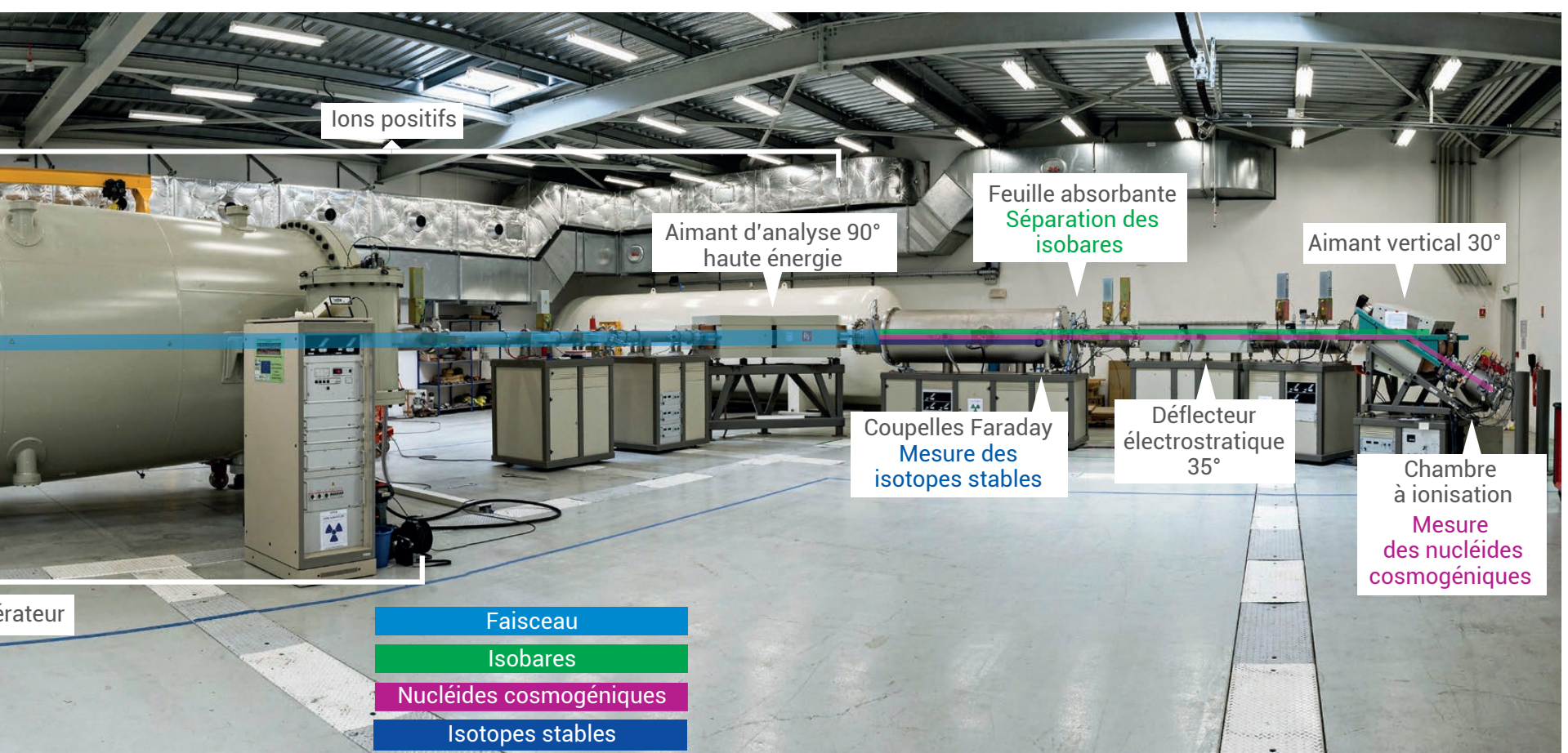

Le spectromètre de masse par accélérateur ASTER

Le spectromètre de masse par accélérateur ASTER (fig. 2) du CEREGE à Aix-en-Provence est équipé de deux sources d'ions acceptant jusqu'à 200 échantillons solides. Ces derniers sont le résultat d'un long processus de séparations et de purifications physico-chimiques, conduisant à partir des différentes matrices complexes échantillonnées (sédiments marins, continentaux et lacustres ; roches granitiques, basaltiques et carbonatées...) à la production de cibles contenant sous la forme d'un composé solide ( $\mathrm{BeO}, \mathrm{Al}_{2} \mathrm{O}_{3}, \mathrm{AgCl}, \mathrm{Agl} \ldots$ ) le nucléide cosmogénique d'intérêt et ses isotopes et isobares surabondants. Pour produire des ions négatifs du nucléide cosmogénique à partir des cibles, du césium (Cs) est introduit sous forme de vapeur dans le corps de source : au contact d'un ioniseur, pièce hémisphérique métallique portée à une température de l'ordre de $120^{\circ} \mathrm{C}$, les atomes de césium sont ionisés en $\mathrm{Cs}^{+}$. La différence de potentiel appliquée entre cet ioniseur et la cible focalise vers cette dernière les ions $\mathrm{Cs}^{+}$qui la pulvérisent. Du fait de leur très grande électropositivité, les atomes neutres de césium transfèrent un électron aux atomes individuels, produisant ainsi des ions négatifs du nucléide cosmogénique d'intérêt.

Extraits de la source grâce à la différence de potentiel appliquée entre la cible et la sortie de la source, les ions négatifs produits sont canalisés par un déflecteur électrostatique, avant d'être analysés en masse à basse énergie par un aimant à $90^{\circ}$. Ils sont ensuite injectés séquentiellement avec un taux de répétition de $\sim 100 \mathrm{~Hz}$ dans l'accélérateur qui délivre une tension terminale maximale de 5 millions de volts (MV). Ils y sont tout d'abord accélérés entre une tension nulle (masse) à l'entrée et la tension maximale appliquée au niveau du terminal (point d'application de la haute tension). Au niveau de ce terminal, ils sont " épluchés " de leurs électrons lors d'interactions avec un faible flux d'argon. Tous les états de charge positifs possibles sont alors produits, mais dans des proportions différentes dépendant de l'énergie communiquée lors de la première étape d'accélération. Ces ions positifs sont accélérés entre la tension terminale et la tension nulle (masse) prévalant à la sortie de la partie accélératrice, d'où l'appellation de «tandem » pour ce type d'accélérateur réalisant des accélérations en deux étapes.

Le spectromètre à haute énergie est doté d'un aimant d'analyse à $90^{\circ}$, auquel est appliqué un champ magnétique dont l'intensité optimise pour la masse du nucléide cosmogénique d'intérêt la transmission le long de son rayon de courbure de l'état de charge positif le plus abondamment produit au niveau du terminal. Les isotopes stables du nucléide d'intérêt suivent des trajectoires internes (pour les plus légers) ou externes (pour les plus lourds) au rayon de courbure de l'aimant et sont collectés sur des coupelles Faraday installées immédiatement après l'aimant d'analyse. Le faisceau, constitué des ions positifs du nucléide cosmogénique d'intérêt et de ses isobares sélectionnés dans l'aimant d'analyse, est envoyé vers une feuille mince $(1 \mu \mathrm{m})$ de nitrure de silicium $\left(\mathrm{Si}_{3} \mathrm{~N}_{4}\right)$ dans laquelle les ions positifs vont perdre une partie de leur énergie initiale, proportionnellement à leur numéro atomique. Les isobares ayant une énergie résiduelle moindre ou supérieure, selon que leur numéro atomique est supérieur ou inférieur à celui du nucléide cosmogénique d'intérêt, sont déviés par un aimant vertical à $30^{\circ}$ et écartés de la trajectoire vers le détecteur final, qui est une chambre d'ionisation à haute résolution à quatre anodes. 
$>>$

plusieurs échelles de temps : variations séculaires directement mesurables par magnétométrie en observatoire et par satellites ; paléovariations séculaires, excursions et inversions restituées par les analyses d'aimantations des roches et des matériaux archéologiques. Ces données doivent être confrontées aux résultats des modèles magnétohydrodynamiques analogiques ou numériques, qui ne peuvent simuler les paramètres géophysiques.

Les mesures du vecteur champ magnétique terrestre réalisées localement en observatoires géomagnétiques ${ }^{(\mathrm{d})}$ et depuis quelques décennies à l'échelle globale par satellites, montrent que le moment dipolaire géomagnétique, dont la valeur actuelle est de $7,810^{22} \mathrm{~A} . \mathrm{m}^{2}$, a chuté de $10 \%$ depuis 1830 . Le relais des données archéomagnétiques ${ }^{(\mathrm{e})}$ indique que cette chute a été amorcée voici presque 3000 ans à partir d'une valeur de l'ordre de $1210^{22}$ A.m². La chute depuis environ 3000 ans atteindrait donc quasiment 30\% de cette valeur.

Les variations de direction et d'intensité restituéesparlesdonnéespaléomagnétiques ${ }^{(\mathrm{e})}$ pour des époques plus anciennes (échelles de $10^{3}$ à $10^{6}$ ans) montrent que le champ magnétique terrestre subit des écarts d'amplitude et de fréquence très variables, probablement contrôlés par différents régimes de la géodynamo. L'étude de l'aimantation rémanente naturelle des roches volcaniques et sédimentaires a permis de démontrer que les inversions de polarité et les excursions sont précédées par une chute du moment dipolaire géomagnétique, suivie d'une annulation du dipôle, avec persistance de sources non dipolaires périphériques [3].

Les données paléomagnétiques présentent toutefois des biais et des incertitudes inhérentes, d'une part aux processus complexes qui gouvernent l'acquisition de l'aimantation rémanente des sédiments et, d'autre part, à la discontinuité temporelle et spatiale des enregistrements d'aimantation par les roches volcaniques. Une approche analytique permet de s'affranchir de ces incertitudes en exploitant la relation de dépendance entre le taux de production des nucléides cosmogéniques dans l'atmosphère et l'intensité du CMT.

Dans le cadre de deux programmes de recherche successifs (ANR Magorb et ERC Edifice), dirigés respectivement par Nicolas Thouveny (CEREGE) et Jean-Pierre Valet (IPGP), l'analyse des concentrations du nucléide cosmogénique ${ }^{10} \mathrm{Be}_{\mathrm{atm}}$ déposé dans des séquences sédimentaires réparties autour du globe terrestre a permis d'établir des séries longues des variations de la composante dipolaire du CMT. Une fois corrigées pour prendre en compte des effets environnementaux résiduels, les séries de ${ }^{10} \mathrm{Be}_{\mathrm{atm}}$ (exprimées en terme de rapport $\left.{ }^{10} \mathrm{Be} /{ }^{9} \mathrm{Be}\right)$ ont été compilées et calibrées sur la base de modèles théoriques et
Grâce à la spectrométrie de masse par accélérateur pour doser les nucléides cosmogéniques radioactifs, il est désormais possible de permettre des études à haute résolution sur des quantités de matière faibles $(\sim 0,1 \mathrm{~g})$, de dater plus précisément sur une gamme temporelle étendue (10 périodes $T_{1 / 2}$ ), de réduire à quelques minutes la durée nécessaire à l'acquisition des résultats, et de simplifier les manipulations lors de la préparation des échantillons pour leur analyse.

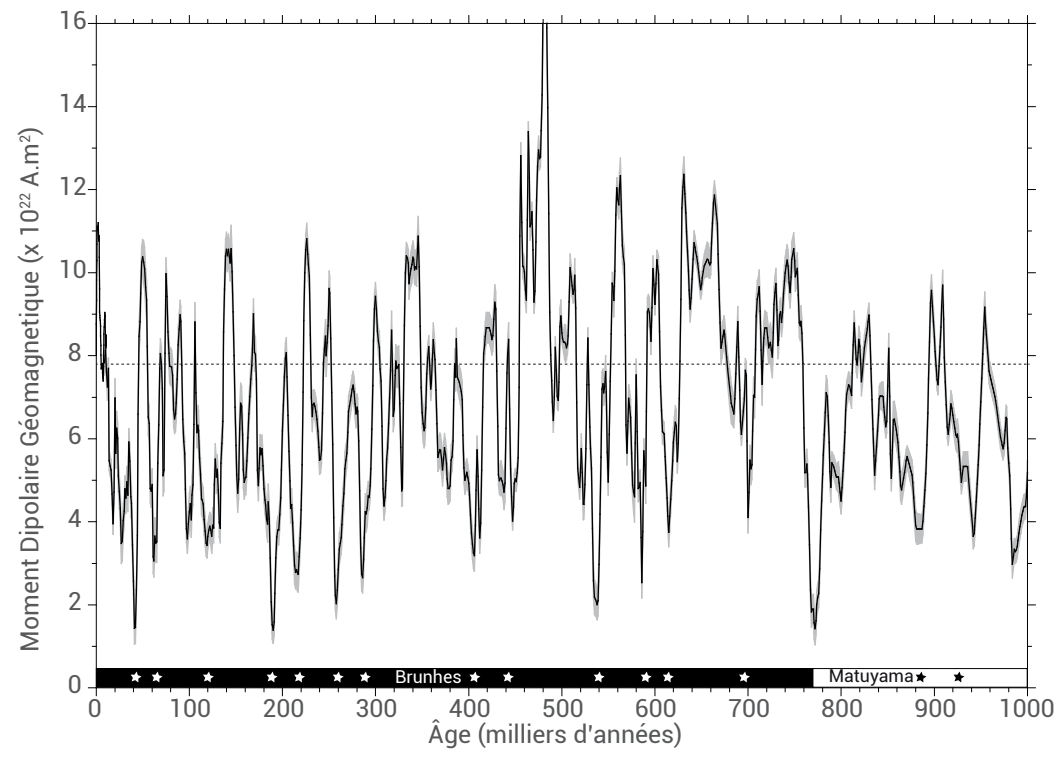

3. Variations passées du moment dipolaire géomagnétique terrestre au cours du dernier million d'années, obtenues à partir d'une compilation calibrée de rapports ${ }^{10} \mathrm{Be} /{ }^{9} \mathrm{Be}[4,5]$. Les lignes de champ du dipôle magnétique terrestre guident les particules chargées du rayonnement cosmique jusqu'en haut de l'atmosphère, où des réactions de spallation génèrent les nucléides cosmogéniques (voir pp. 16-17). La production de béryllium 10 dépend de la pénétration du rayonnement cosmique dans la magnétosphère et de l'orientation des lignes de champ magnétique. Cette production est plus faible aux basses latitudes, où les particules chargées sont défléchies par les lignes de champ, mais cet effet de latitude est effacé par la circulation atmosphérique. Les évènements d'inversion ou d'excursion du champ magnétique terrestre, où ce champ est faible, laissent donc plus passer les rayons cosmiques et se traduisent par des pics de concentration du béryllium 10, 
$>>$

empiriques de production de cet isotope, afin d'obtenir une courbe de référence qui met en évidence l'extrême variabilité du moment dipolaire géomagnétique en termes d'amplitude et de fréquence au cours du dernier million d'années (fig. 3). L'observation la plus remarquable est la succession de plus d'une quinzaine d'épisodes de surproduction du ${ }^{10} \mathrm{Be}_{\text {atm }}$ (jusqu'à un doublement pour certains évènements), contrôlés par des effondrements ponctuels du moment dipolaire géomagnétique. Un de ces épisodes correspond à la dernière inversion de polarité survenue il y a 773000 ans (la transition MatuyamaBrunhes), mais de nombreux autres caractérisent les chutes d'intensité liées à des excursions dont l'occurrence était plus ou moins bien révélée par les données paléomagnétiques.

La valeur actuelle du moment géomagnétique est égale à la valeur moyenne restituée pour le dernier million d'années, donc encore loin des valeurs critiques associées aux déclenchements d'excursions (de l'ordre de 20 à 30\% du champ actuel). Cette observation suggère que la décroissance actuelle du champ s'inscrit dans le cadre d'une variation démarrée dans un contexte de champ particulièrement fort. Cependant, lorsque l'on aborde le taux de décroissance moyen restitué par les données archéomagnétiques, on constate qu'il est équivalent, voire supérieur, aux taux de variations qui ont précédé les excursions et inversions passées. Cette observation suggère qu'après 773000 ans de polarité normale, et une quinzaine de tentatives d'inversions ayant abouti à de brèves excursions, dont la dernière a eu lieu voici 41000 ans (excursion Laschamp), la conservation d'un tel taux de décroissance aboutirait en moins de 2000 ans au seuil de déclenchement d'une excursion ou d'une inversion.

L'étude de séquences sédimentaires déposées très rapidement durant des évènements similaires est aujourd'hui indispensable pour reconstruire précisément les variations rapides du champ qui ont précédé ces excursions et inversions. Ces données sont essentielles pour contraindre le spectre des variations temporelles géomagnétiques et tenter d'améliorer la compréhension des processus géophysiques qui les engendrent. Elles permettent aussi de soutenir les simulations numériques, et d'anticiper l'évolution future du champ magnétique terrestre.

\section{Conclusion et perspectives}

Une des avancées les plus remarquables liée au développement de la technique de spectrométrie de masse par accélérateur est l'amélioration par un facteur un million à un milliard de la sensibilité de détection, comparée à celles des techniques classiques et pour l'ensemble des nucléides cosmogéniques étudiés jusqu'à ce jour. Cette amélioration a de nombreuses conséquences, qui sont encore loin d'être exploitées à leur pleine mesure.

À titre d'exemple, le dosage des nucléides cosmogéniques produits dans la matrice des minéraux exposés au rayonnement cosmique, notamment le ${ }^{10} \mathrm{Be}$ et le ${ }^{36} \mathrm{Cl}$, permet d'établir la chronologie des évènements climatiques en domaine continental, de contraindre la vitesse des déformations tectoniques et donc d'évaluer l'aléa sismique. L'étude de la variation de leur concentration le long de profils d'altération (profils le long desquels les propriétés physico-chimiques des minéraux sont

(a) Anomalie de l'Atlantique Sud : il s'agit d'une zone, centrée sur la côte sud du Brésil, où le champ magnétique terrestre $(\sim 25 \mu \mathrm{T})$ est nettement plus faible que sa valeur moyenne $(\sim 40 \mu \mathrm{T})$, ce qui s'explique par le fait que la partie interne de la ceinture de Van Allen y est plus proche de la Terre.

(b) Inversions de polarité : épisodes durables, qui surviennent occasionnellement lors de phases d'effondrement de l'intensité du champ magnétique terrestre (le pôle nord magnétique que l'on connait actuellement s'est par exemple établi il y a 773000 ans, alors qu'il se situait dans l'hémisphère Sud auparavant).

(c) Excursions : épisodes rapides d'instabilités directionnelles lors de phases d'effondrement de l'intensité du champ magnétique terrestre, suivis par un retour à la polarité initiale, contrairement aux inversions.

(d) Un observatoire géomagnétique mesure en continu le champ magnétique terrestre. L'Observatoire Magnétique National, qui dépend de l'Institut de Physique du Globe, est situé à Chambon-la-Forêt (45). Il comprend un magnétomètre vectoriel, qui mesure les trois composantes et donc l'orientation du champ, et un magnétomètre scalaire absolu, qui mesure la force totale du champ. Les données sont enregistrées une fois par minute.

(e) Le paléomagnétisme est la discipline qui étudie le champ magnétique terrestre passé. L'archéomagnétisme est l'étude des traces du champ magnétique terrestre dans les objets archéologiques.

(f) Abiotique : qui n'implique aucune réaction biologique (au contraire de "biotique"). modifiées par des réactions abiotiques ou biotiques $^{(f)}$ ) permet de comprendre les mécanismes de mise en place et de développement des sols, et de quantifier les taux de dénudation et d'accumulation de surfaces actives et reliques. Ils offrent également un moyen unique pour mesurer l'impact des activités humaines sur les taux de dénudation. Le dosage du chlore 36 atmosphérique est aussi particulièrement adapté à l'étude de l'évolution des aquiferes et constitue donc un puissant outil d'aide à la gestion de l'eau, un enjeu majeur des années à venir ; il permet de préciser les mécanismes de recharge en eau, de déterminer la vitesse de déplacement des eaux artésiennes et d'évaluer le temps de résidence de l'eau souterraine dans différents réservoirs. Enfin, le béryllium 10 est un traceur et géochronomètre qui donne accès à des plages temporelles s'étendant jusqu'à il y a environ $14 \mathrm{Ma}$ pour la seule variété atmosphérique, et environ $5 \mathrm{Ma}$ pour la variété in situ combinée avec la variété in situ de l'aluminium 26.

Références

1. J. Beer et al., Cosmogenic Radionuclides - Theory and Applications in the Terrestrial and Space Environments, Springer-Verlag, Berlin (2012).

2. A.-E. Lebatard et al., "Cosmogenic nuclide dating of Sahelanthropus tchadensis and Australopithecus bahrelghazali: Mio-Pliocene hominids from Chad", PNAS 105 (2008) 3226-3231.

3. J.-P. Valet et A. Fournier, "Deciphering records of geomagnetic reversals", Review of Geophysics 54 (2016) 410-446.

4. Q. Simon et al., "Authigenic ${ }^{10} \mathrm{Be} /{ }^{9} \mathrm{Be}$ ratio signatures of the cosmogenic nuclide production linked to geomagnetic dipole moment variation since the Brunhes/Matuyama boundary", J. Geophys. Res. Solid Earth 121 (2016) 7716-7741.

5. Q. Simon et al., "Cosmogenic signature of geomagnetic reversals and excursions from the Réunion event to the Matuyama-Brunhes transition (0.7-2.14 Ma interval)", Earth Planet Sci. Lett. 482 (2018) 510-524. 